\title{
eMBeDding for impact and scale in developing contexts
}

\author{
VARUN GAURI*
}

\begin{abstract}
While behavioral public policy remains underutilized in rich countries relative to its potential impact, the required infrastructure for diagnosing behavioral bottlenecks and the autonomy to act on those diagnoses are even less common in developing countries. At the World Bank, the Mind, Behavior, and Development Unit (eMBeD) aims to promote the systematic use of behaviorally informed tools in development policies and projects, institutionalize the use of behavioral science in development organizations and governments, provide evidence on scaled and sustainable behavioral solutions, and generate more and better behavioral data. By focusing on thorough diagnosis and ongoing adaptation, we aim to create impactful, behaviorally informed interventions in complex, resourceconstrained settings. While Sanders, Snijders and Hallsworth (2018) raise valuable points about the implications for impactful behavioral public policy, for those working in developing countries, issues of replication and scale come with unique contextual challenges.
\end{abstract}

Submitted 11 January 2018; accepted 12 March 2018

The UK Behavioural Insights Team (BIT) has been a pioneer in the application of recent research in the behavioral sciences to policy-making. It is impossible to attend a conference on the topic without several speakers appreciating and recognizing its contributions. In their article, Sanders, Snijders and Hallsworth (2018; henceforth SSH) note that BIT has now 'spun off' its governance. It is also true that BIT has spun around the globe, with activities in every continent. And it has spun off new business lines, which now include the development of software and other platforms to facilitate 'nudging' and behavior change, as well as the advocacy and use of evidence in policy-making more generally. To many of us working in the field, including the staff of the World

* Correspondence to: Varun Gauri, Co-Head, The Mind, Behavior, and Development Unit, The World Bank, Washington, DC, USA. Email: vgauri@worldbank.org 
Bank, BIT remains an important inspiration, standard-bearer, trend-setter and partner.

As the authors mention, the World Bank now has a dedicated team working in the area - the Mind, Behavior, and Development Unit (eMBeD). ${ }^{1}$ Our objectives are to promote the systematic use of behaviorally informed tools in development policies and projects, institutionalize the use of behavioral science in development organizations and governments, provide evidence on scaled and sustainable behavioral solutions, and generate more and better behavioral data.

We accomplish those objectives by working closely (embedding) with policymakers in their ongoing projects and by conducting fieldwork. Our approach, like that of BIT and our other partners, is to identify bottlenecks and then design and evaluate behaviorally informed interventions to address them. We also focus on building capacity through advisory services and workshops and collaborate with partners internal and external to the World Bank in the process. In short, we use behavioral science to diagnose, design, implement and evaluate development projects and programs, with the goal of ending global poverty and enhancing equity.

Because our mandate is global, we encounter governments at varying levels of capacity and technical sophistication. We also confront an extremely broad range of problems to tackle. As a result, our perspective may differ somewhat from that described by SSH.

I agree with the broad directions of SSH's review of the state of the field. SSH do, however, emphasize the challenges to behavioral science more than the obstacles facing those of us working in behavioral public policy. For some of the problems they address, such as the replication crisis, the implications of cultural diversity, and reverse impact, our colleagues in academia may be better placed to develop assessments and solutions. Those of us working in behavioral public policy, including the eMBeD team, certainly have things to say on each of these issues. But I think our contributions and reflections are strongest in the domain of public policy. In what follows, I start with the policy challenges. Then I turn to some of the conceptual and data issues they raise.

\section{Development and behavioral public policy}

They key idea in our field is that decision-making and behavior are strongly influenced by contextual variables that standard policy analysis tends to omit. This is a universal problem, but it can be mitigated, and richer countries have often developed, perhaps unintentionally or organically, approaches for 
doing so. One of these is delegation and decentralization. In several highincome countries, the implementation of service delivery is often delegated to local governments, technical professionals and frontline service providers. These actors are often in a better position to identify contextual variables, and decentralization empowers them to modify details of implementation so as to take account of them. For instance, long before BIT arrived, many teachers had developed more participatory and collaborative pedagogical styles because they believed that their students learn better when the curricular content is more 'social' and engaging. In many developing countries, topdown teaching styles prevail due to civil service rules, the absence of decentralization and a lack of capacity at the local level. More generally, the lack of autonomy and capacity make it difficult to tailor service delivery to the context.

At the same time, in richer countries, technology is increasingly allowing service users and consumers to modify implementation and delivery processes. For instance, mobile banking customers can now set up their own reminders to make loan repayments and even enroll in automated transfers so that they do not miss their payment dates. Technology is facilitating self- nudging. These technologies remain incipient in many developing countries.

Of course, behavioral public policy remains, relative to its potential impact, underutilized in rich countries, as the demand for the work of BIT and behavioral insights units has shown. Still, the organizational and technical infrastructure for diagnosing contextual obstacles to desired behaviors and the autonomy to act on those diagnoses are even less common in developing countries. That makes the case for behavioral public policy in developing countries stronger.

But this has implications not only for the value of behavioral public policy in developing countries, but also for where its payoffs might be biggest. Diagnosing contextual variables that affect implementation is important. However, even if a developing country's government has the capacity and autonomy to implement changes in one implementation context, it may lack the power to do so in another, or may lose that autonomy in the future, because local-level capacity and autonomy tend to be constrained.

The larger payoff for behavioral development policy may derive from bringing behavioral diagnostics upstream, to the stage of policy design and regulation. For a variety of reasons, including the preference for randomized evaluation in the field of behavioral public policy, much of the work remains at the stage of project implementation. Randomized evaluation is important, of course, and, up to a point, it is reasonable for funders to want to measure the impacts of their contributions. But I would argue (though of course this would have to be backed up by data) that the field of behavioral public policy could also benefit from the regular input of behavioral science earlier, 
at the stage of program design and regulation. This kind of analysis is consistent with recent efforts to pay attention to context in policy design and to move away from 'cut and paste' projects and toward iterative and adaptive programming (see World Bank, 2015, Chapter 9; Andrews et al., 2013). For a variety of reasons, including the preferences of donors, contextual and adaptive policy design remains the exception rather than the rule.

Behavioral development policy also addresses challenges that often differ from the demands that behavioral teams in high-income countries typically face. To provide just a few examples, at $\mathrm{eMBeD}$, we are working on the promotion of breastfeeding, the take-up of off-grid micro-solar panels and biogas digesters, improving parenting practices on the part of low-literacy parents, increasing latrine use, improving social cohesion, and getting women into the workforce in countries where that is not the norm. It is clear that behavioral public policy is valuable for developing countries; at the same time, developing countries are valuable for behavioral public policy because they present new challenges that can illuminate as-yet untested interventions in new domains. If you want to 'nudge for good', there may be no better place to work than in low-income countries.

\section{Developing countries and behavioral science}

A central idea of behavioral public policy is 'libertarian paternalism' - the idea that nudges should be costless or easy to reverse if individuals determine that they are not in their best interests (Thaler \& Sunstein, 2008). That notion is not uncontroversial, as there is an asymmetry that is unavoidable - if you believe that inertia, myopia and other biases are inherent in human nature and that is why nudges are valuable, reversing a nudge - say, opting out of a retirement savings plan you have been defaulted into - may be costlier than one expects (Gauri, forthcoming 2018). Still, it remains a guiding principle for a significant amount of work in the field.

In developing countries, however, many of the most pressing concerns involve social norms, mental models of social groups and obstacles to collective action. For instance, in places 'where corruption is the norm', changing that norm may leave certain individuals worse off (World Bank, 2015). Similarly, efforts to change social norms in favor of gender equality may reduce leisure time for men, as they now have to contribute more time to household chores. In other words, in the development context, the non-libertarian aspects of the behavioral insights agenda become more prominent, and it is difficult to make do without a substantive account of social welfare. In our own case at the World Bank, we take our bearings from the institutional mission to eliminate global poverty and enhance equity. 
SSH note the challenge of the 'replication crisis' in social psychology for behavioral insights work. It is certainly true that some widely cited findings may not hold up when implemented in new contexts. For instance, the idea that priming potential taxpayers with social norms is an extremely effective nudge emerged from BIT's work and has been replicated in contexts as different from the UK as Guatemala. But our recent work in Poland showed that 'hard tone' letters were more effective than social norms priming (Hernandez et al., 2017).

The replication crisis point is related to the difficulty of finding 'true universals' given cultural variation worldwide. That is a problem that we encounter regularly at the World Bank. I do not think, however, that the appropriate response is to have new behavioral insights teams 'conduct very similar replication studies'. Cultural variation is primarily a problem for academics testing whether psychological mechanisms are similar across societies. When it comes to economic outcomes, moreover, the important source of variation is likely not cultural but organizational. Culture, in the sense of the schema and decision-making frames that individuals bring to bear on a problem, is more relevant than the larger macro-culture, which is often treated as a black box, in any case (DiMaggio, 1997). For example, as Fukuyama put it sometime ago, "a large cultural variable like Catholicism may not be very helpful in explaining or predicting political behavior, but a norm which assumes that bureaucratic appointments ought to favor friends and relatives over people with formal credentials might" (Fukuyama, 2007). Finally, there is no way to be sure that an intervention will work, even if it has been shown to work in that very context, because something working at time $t$ does not mean it will work at time $t+1$ (Cartwright \& Hardie, 2012).

Our approach to this problem is to conduct careful diagnostics, whenever possible, before and during the implementation of an intervention, and then to adapt that intervention in an ongoing manner. In this approach, one focuses less on proving that something worked in context $i$ and at time $t$, and more on whether the premises on which the policy was based were scientifically sound. When interventions are based on good diagnostics, which include survey experiments, $\mathrm{A} / \mathrm{B}$ testing and ethnographic work, among many other methods, we have reason to believe that the interventions had a sound foundation. Not every sound intervention works, and behavioral policy should of course endeavor to assess whether its interventions are making a difference, but requiring a measure of the impact of every single intervention is likely too costly, given the benefits.

Of course, not every policy intervention lends itself to a randomized controlled trial (RCT). Policy-level and regulatory changes, which SSH endorse and are crucial in development, as I suggested above, are not easy to evaluate 
with RCTs. They should be evaluated when doing so makes sense, but more importantly, they should be grounded in sound science.

To SSH's list of mechanisms through which nudges can lead to 'long-term effects' I would add the impact of small changes in 'self-reinforcing recursive processes'. There are key junctures in life - before getting tracked into a higher or lower curriculum, early childhood development and the early years of working - and small changes in those moments can have lifelong consequences because they affect a broader field of forces propelling people forward or holding them back (Yeager \& Walton, 2011). Small socialpsychological changes can boost a person's chances by putting them on a different trajectory (college preparatory or not) or because they help one acquire human capital that can be put into the service of the acquisition of further human capital - 'skills beget skills' (Heckman, 2008).

On scaling, I entirely agree that implementation at scale is the ultimate goal in behavioral public policy, that this has been a shortcoming in the field and that, as SSH put it, "academic incentives encourage innovative results and highly cited publications, but fail to encourage researchers to scale their interventions." There may be insights into homophily, as the authors put it - or institutional isomorphism, to use an older term (Meyer \& Rowan, 1997) that behavioral science and organizational psychology can contribute to, and in so doing help us to understand the obstacles to scaling. But I think the more fundamental problem is that behavioral public policy has been too focused on RCTs and trials, at the expense of moving earlier in the cycle into policy design. There is much to be done on regulation, for instance, and showing how behavioral insights can contribute would help make the case for its systemic application. Moving earlier in the project cycle would not necessarily require a BIT-type team embedded in every ministry. In the same way that a planning ministry does cost-benefit analyses for regulations and projects, the ministry could also conduct behavioral assessments of the same, perhaps even as a standard component of cost-benefit analyses.

I also agree that organizational effectiveness is an important target for behavioral public policy (Banuri et al., 2017), and it is an important route for achieving impact at scale. To my eye, the main obstacle is disciplinary. Social and cognitive psychologists, alongside behavioral economists, have been, by and large, the main protagonists in behavioral public policy so far. But organizational effectiveness work will require collaborations with political scientists, organizational scientists, sociologists, anthropologists and others. The methods of analysis will be different, as will the key variables affecting decision-making. This is an important frontier for behavioral public policy, and behavioral insight teams around the world may ourselves need to adapt to address it. 


\section{References}

Andrews, M., L. Pritchett, and M. Woolcock (2013), 'Escaping Capability Traps through ProblemDriven Iterative Adaptation (PDIA)', World Development, 51(Nov.): 234-44.

Banuri, S., S. Dercon and V. Gauri (2017), Biased policy professionals (English). Policy Research working paper; no. WPS 8113. Washington, D.C.: World Bank Group.

Cartwright, N. and J. Hardie (2012), Evidence-Based Policy: A Practical Guide to Doing It Better, New York: Oxford University Press.

DiMaggio, P. (1997), 'Culture and cognition', Annual Review of Sociology, 23: 263-287.

Fukuyama, F. (2007), 'Development and the Limits of Institutional Design', in Natalia Dinello and Vladimir Popov, (eds), Political Institutions and Development: Failed Expectations and Renewed Hopes, Cheltenham, UK: Edward Elgar.

Gauri, V. (forthcoming 2018), 'Nudging Goes Global: Comments on 'Behaviorally Informed,' by Cass Sunstein', in Kaushik Basu (ed.), The State of Economics, The State of the World, MIT Press.

Heckman, J. J. (2008), 'Schools, Skills, and Synapses', Economic Inquiry, 46(3): 289-324.

Hernandez, M. A., J. C. Jamison, E. J. Korczyc, N. Mazar and R. C. Sormani (2017), Applying behavioral insights to improve tax collection: experimental evidence from Poland (English), Washington, D.C.: World Bank Group.

Meyer, J. W. and B. Rowan (1977), 'Institutionalized organizations: Formal structure as myth and ceremony', American Journal of Sociology, 83: 340-363.

Sanders, M., V. Snijders and M. Hallsworth (2018), 'Behavioural science and policy: where are we now and where are we going?', Behavioural Public Policy, 2(2): 144-167.

Thaler, R. H. and C. R. Sunstein (2008), Nudge: Improving Decisions about Health, Wealth, and Happiness, New Haven, CT: Yale University Press.

World Bank Group (2015), World Development Report 2015: Mind, Society, and Behavior, Washington, DC: World Bank.

Yeager, D. S. and G. M. Walton (2011), 'Social-Psychological Interventions in Education: They're Not Magic', Review of Educational Research, 81(2): 267-301. 